\title{
Fully Integrated Seven-Order Frequency-Range Quadrature Sinusoid Signal Generator
}

\author{
Chao Yang and Andrew J. Mason, Senior Member, IEEE
}

\begin{abstract}
A compact wide-frequency-range quadrature sinusoid signal generator tailored to on-chip impedance spectroscopy instrumentation applications is described. Using a new hybrid structure, it provides seven orders of frequency-tuning range (1 $\mathrm{mHz}$ to $10 \mathrm{kHz}$ ) appropriate for impedance characterization of many sensor materials. The signal generator exhibits very accurate frequency tuning through digital control, and the new structure inherently guarantees good phase and amplitude matching between sine and cosine outputs across the supported frequency range. The circuit has been fabricated in a $0.5-\mu \mathrm{m}$ CMOS process and occupies $1 \mathrm{~mm}^{2}$. It consumes only $60 \mu \mathrm{A}$ with a $3-\mathrm{V}$ supply. Measurements show that the phase mismatch is less than $0.8^{\circ}$ and that the amplitude mismatch is less than $3 \%$. The total harmonic distortion is below $0.6 \%$ over the seven-order frequency range.
\end{abstract}

Index Terms-Analog filter, impedance measurement, quadrature signal generator, sinusoid signal generators, wide-frequencyrange frequency tuning.

\section{INTRODUCTION}

$\mathbf{Q}$ UADRATURE sinusoid signals are two sinusoid signals with the same frequency and amplitude but with a $90^{\circ}$ phase difference. They are necessary for signal processing in many applications. To suppress image channel interference in wireless communication systems, quadrature sinusoid signals enable complex signal processing with in-phase and quadrature-phase signals. In instrumentation applications, they are used to extract impedance/admittance information. Some applications require only square-wave quadrature signals [1], whereas applications with tighter accuracy constraints demand high-quality continuous-time quadrature sinusoid signals to guarantee performance. Typically, the quality of a quadrature sinusoid signal is measured by the frequency accuracy, linearity, and phase/amplitude matching between the two signal outputs.

This paper introduces a quadrature sinusoid signal generator (QSSG) designed for a low-frequency on-chip impedance spectroscopy (IS) instrument that is suitable for characterizing a wide range of sensor interfaces. The frequency range of the IS instrument, and therefore the QSSG, was broadened to meet the low-frequency requirements of biochemical sensors based on biomimetic interfaces with membrane proteins [2], [3]. The

Manuscript received February 27, 2008; revised June 10, 2008. First published August 21, 2009; current version published September 16, 2009. This work was supported by the Engineering Research Centers Program of the National Science Foundation (NSF) under Award Numbers EEC-9986866 and DBI-0649847. The Associate Editor coordinating the review process for this paper was Dr. Izzet Kale.

C. Yang was with the Department of Electrical and Computer Engineering, Michigan State University, East Lansing, MI 48824-1226 USA. He is now with Marvell Semiconductor, Inc., Santa Clara, CA 95054 USA (e-mail: yangchao@ msu.edu).

A. J. Mason is with the Department of Electrical and Computer Engineering, Michigan State University, East Lansing, MI 48824-1226 USA.

Digital Object Identifier 10.1109/TIM.2009.2018001

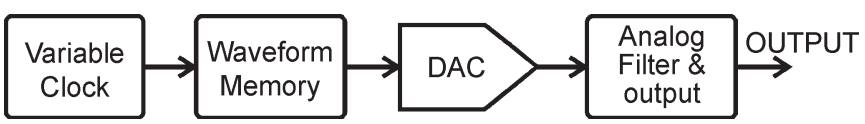

Fig. 1. Block diagram of a DSP-based sinusoid signal generator.

resulting challenge is providing a tunable frequency range from $1 \mathrm{mHz}$ to $10 \mathrm{kHz}$, seven decades, with good frequency accuracy and phase/amplitude matching between sine and cosine outputs. Circuit size is also a critical constraint in the QSSG design to support the implementation of a fully on-chip IS instrument suitable for sensor arrays.

Many methods for sinusoid signal generation can be found in commercial products and research papers. Generally speaking, these methods can be categorized into three groups, namely, DSP-, oscillator-, and nonlinear-circuit-based solutions.

DSP-based solutions generate a sinusoid signal using digitally stored information [4], [5]. As shown in Fig. 1, they consist of a digital memory bank, a digital signal processor, a digital-to-analog converter (DAC), and analog output circuits. Frequency turning is achieved by different strategies [6] to vary the memory updating clock. They are very flexible, and nearly all waveform shapes can be obtained by changing the memory bank contents. However, their value becomes limited at very low frequencies. To relax the design requirements of the analog filter, the update clock frequency cannot be very low. Thus, a very high signal oversampling ratio (OSR) is expected for low-frequency outputs. To store the waveform for each sampling point over the whole cycle, prohibitively large memory is needed. Although this memory is easily achievable with modern IC technology, the size of the memory would bar implementation of a compact signal generator for on-chip IS instrumentation.

Oscillators are the most widely used circuits to generate pure sine waves. Quadrature oscillators have also been reported for generating quadrature signals [7], [8]. In an oscillator-based sinusoid signal generator, the frequency is controlled by a tunable component in the circuit, such as a resistor, a capacitor, or a transconductor. The limitation with these oscillatorbased signal generators lies in their narrow frequency-tuning range; the tuning component values, which control the output frequency, cannot be controlled over a very wide range (seven to eight orders, as desired). Although a subthreshold tunable CMOS transconductance that provides a frequencytuning range over seven decades has been reported [9], the high-frequency accuracy is limited because it is impractical to precisely tune the transconductance over that wide of a range.

In nonlinear-transfer-function-based signal generators [10], a nonlinear transformation is utilized to convert the triangle wave to a sine waveform, as shown in Fig. 2(a). MOS transistors can 


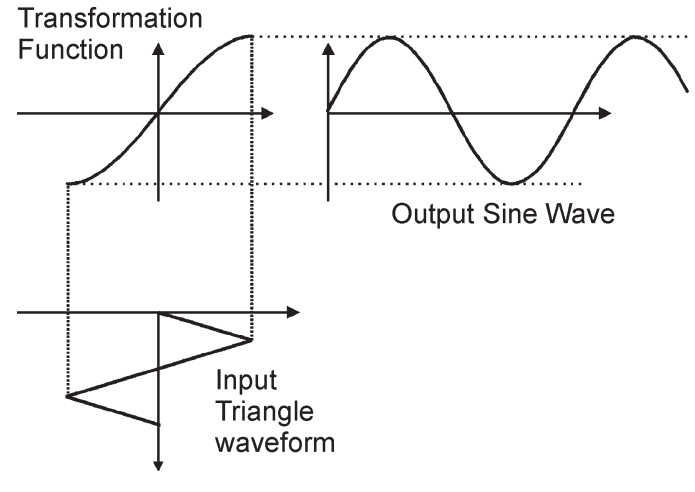

(a)

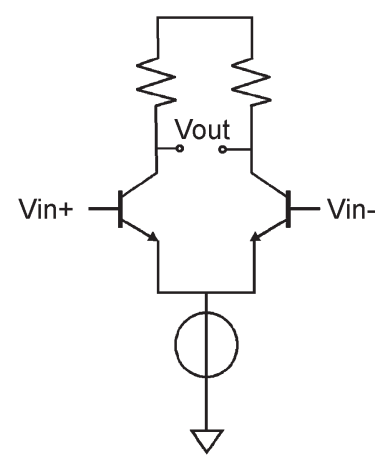

(b)

Fig. 2. Principles of the triangle-to-sine signal generator. (a) Transformation from a triangle waveform to a sine waveform. (b) Typical circuit performing the transformation.

also be used to realize the nonlinear functions [11]. Because a triangle wave can easily be generated, these nonlinear-transferfunction-based methods provide a simple means of realizing a sinusoid signal generator. However, the main drawback of the triangle-to-sine generator is its linearity, which is limited by how well the nonlinear function approximates half of the sine wave. In addition, both the peak value and the commonmode bias voltage of the triangle wave are very critical to the quality of the sinusoid output. For on-chip applications, the nonlinear transfer function is adversely affected by process and temperature variations of semiconductor devices, making it hard to control its quality in terms of linearity. Furthermore, if this type of generator were employed for quadrature outputs, two identical nonlinear transfer functions would be required, and any mismatch between them would directly contribute to the mismatch between sine and cosine outputs.

All of these traditional signal generators are not suited to construct a compact QSSG circuit covering from $1 \mathrm{mHz}$ to $10 \mathrm{kHz}$ due to their limitations in frequency range, physical size, or quality of the waveform. In this paper, a new hybrid structure QSSG was designed and tested. The new circuit can generate quadrature sinusoid signals covering from $1 \mathrm{mHz}$ to $10 \mathrm{kHz}$ with inherently good phase $\left(0.8^{\circ}\right)$ and amplitude matching (3\%). Section II describes the architecture of the new signal generator. Its VLSI realization is presented in Section III, and measurement results of the fabricated circuit are provided in Section IV. Section V gives conclusions.

\section{New Architectures AND PRinciples}

Of the aforementioned traditional signal generator topologies, none are well suited to construct a compact QSSG circuit covering from $1 \mathrm{mHz}$ to $10 \mathrm{kHz}$ due to their limitations in frequency range, physical size, or quality of the waveform. To overcome these limitations, a new architecture is introduced here that can generate high-quality outputs over seven decades of the frequency range. The new signal generator topology employs a hybrid architecture, integrating two different structures for low- and high-frequency outputs. It is purely controlled by digital signals, and its output frequency is set with respect to a single master clock to achieve high-frequency accuracy. A system-level view of the proposed signal generator is shown in Fig. 3.

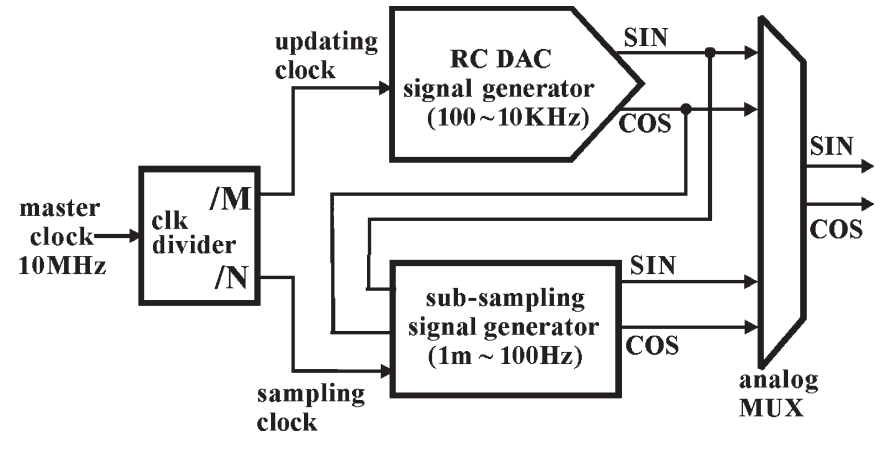

Fig. 3. Principle schematic of the new hybrid quadrature signal generator.

For the frequency range between $100 \mathrm{~Hz}$ and $10 \mathrm{kHz}$, a signal generator based on a resistor-chain DAC (RCDAC) is employed to generate the quadrature sinusoid signals. It provides exact frequency control for the output sinusoid signal by controlling the frequency of a digital updating clock. It also guarantees good matching between output sine and cosine signals because both of them are generated from the same resistor chain.

To generate low frequencies $(1 \mathrm{mHz}$ to $100 \mathrm{~Hz})$, the outputs of the RCDAC signal generator are subsampled, i.e., sampled at lower frequencies. To our knowledge, this is the first time that the subsampling technique has been utilized in signal generators. As will be detailed later in this paper, this subsampling signal generator provides very good coverage for low frequencies with a minimal chip area.

As will be detailed in Section III-C, both circuit paths will require low-pass filters (LPFs) at their outputs to suppress the replicas and remove high-frequency switching glitches. It will be shown that the RCDAC requires at least $17-\mathrm{dB}$ replica suppression, whereas the subsampling circuit requires up to 13-dB replica suppression.

\section{A. QSSG Based on a Sinusoidally Tapped RCDAC for $100 \mathrm{~Hz}$ to $10 \mathrm{kHz}$}

For high-frequency (100 Hz to $10 \mathrm{kHz}$ ) sinusoid signals, an RCDAC is unevenly tapped so that its output steps a sinusoid waveform. The sinusoid output is generated by sequentially activating these taps at a rate determined by the master clock signal. A simplified example of the RCDAC, as shown in Fig. 4, illustrates this operation principle. Here, by sampling 


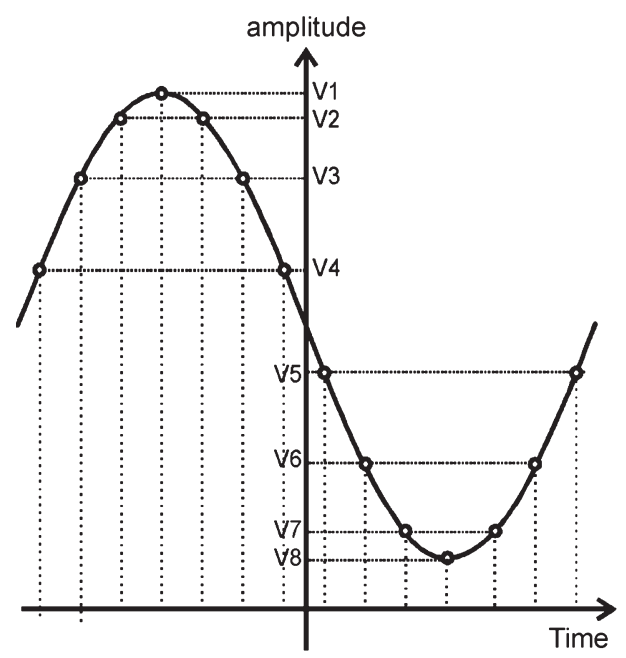

(a)

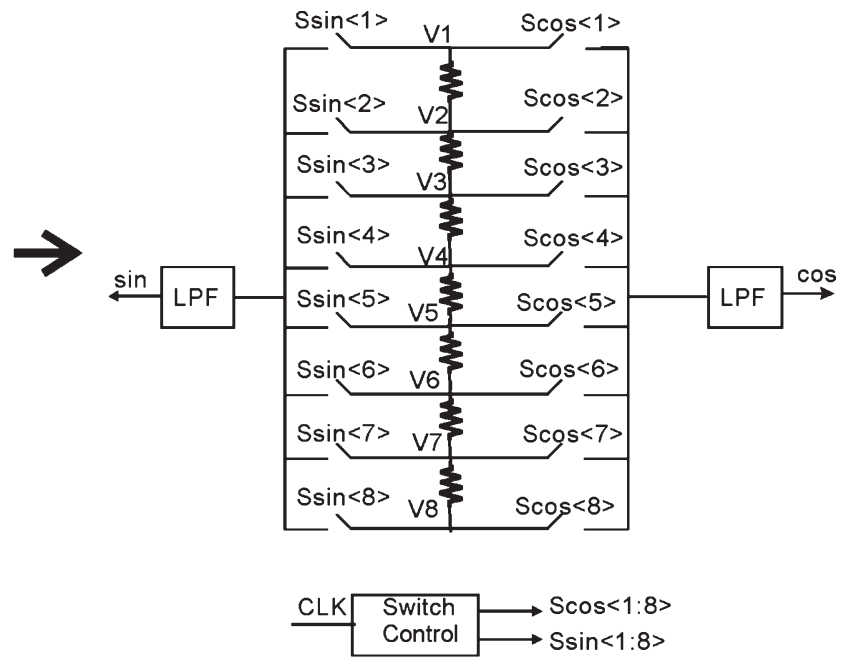

(b)

Fig. 4. Principles of a sinusoidally tapped RCDAC quadrature sinusoid signal generator. A resistor chain with only eight taps is demonstrated for illustration; the real system has 33 taps.

one period of the sinusoid at 14 regular intervals, the eight sample points for a sine wave [Fig. 4(a)] can be stored in only eight DAC taps [Fig. 4(b)] by setting appropriate values for resistors in the chain. The top and bottom voltages of the resistor chain determine the peak and valley of the sinusoid wave, respectively. To simultaneously generate cosine and sine waveforms, a separate set of switches is employed on each side of the chain and activated out of phase. At any time, only one switch on each side is turned on to pick the appropriate tap in the chain. The sequence of turning on the switches is 1-2-3-4-5-6-7-8-7-6-5-4-3-2, and the frequency is determined by the updating clock. By periodically repeating this sequence, a sinusoid waveform is generated. The sequence is the same for both sine and cosine outputs, except that switch activation is offset by four switches (in this example) to introduce a $90^{\circ}$ phase difference. An LPF is included at the DAC output to smooth the output waveform and filter out replicas. Because both sine and cosine waveforms are generated from the same resistor chain, quadrature matching is very good.

Clock jitter in the digital control circuitry will introduce variations in the switching time of the RCDAC. Based on reported jitter values [12], this variation should only be on the order of picoseconds. Because the maximum output frequency of the signal generator is $\sim 10 \mathrm{kHz}$, this clock jitter should have a negligible effect.

Comparing this RCDAC-based sinusoid signal generator with that based on the DSP techniques briefly reviewed in Section II [4], [5], we see that both utilize an updating clock to generate the sine wave and control the frequency. However, in our new design, the "sinusoidally coded" RCDAC replaces the waveform memory and general DAC in Fig. 1, thus eliminating the need for digital memory and saving a significant silicon area. Similar to its DSP counterpart, the RCDAC-based QSSG cannot generate a very low frequency because the number of taps available in the R-chain, representing its "memory size," is limited by physical routing and other practical issues.

In the current implementation of the RCDAC-based highfrequency QSSG, we have set the number of taps to 33, which corresponds to a $64 \times$ OSR. The lowest output frequency is set to $100 \mathrm{~Hz}$, where the low-frequency sinusoid signal generator (see succeeding discussion) takes over.

\section{B. Subsampling QSSG for $1 \mathrm{mHz}$ to $100 \mathrm{~Hz}$}

In contrast to Nyquist sampling techniques, where the sampling frequency is required to be more than twice the signal frequency, the subsampling technique relies on a sampling frequency that is less than twice the signal frequency. The sample-and-hold results have a fundamental signal and many replicas in the frequency spectrum. The desired output is one of the replicas. This technique is often used in communication systems to down convert the information from radio to low intermediate frequency, enabling signal processing [13]. In our design, a novel sinusoid generator topology based on the subsampling concept was developed to produce very low frequency outputs.

The "reference" inputs to the subsampling signal generator are the quadrature sinusoid signals produced by the RCDACbased signal generator. These signals are sampled at a frequency that is very close to the signal frequency, i.e., the frequency difference is very small. The output of the sampling circuit contains a low-frequency replica that represents the frequency difference between the input signal and the sampling clock. This replica can be extracted to obtain the desired lowfrequency sinusoid outputs. The sequence of a sampled sinusoid input is given by

$$
f(n)=\sin \left(2 \pi f_{0} \frac{n}{f_{s}}\right)
$$

where $n$ is the index of the discrete sequence, $f_{0}$ is the signal frequency, and $f_{s}$ is the sampling frequency. Assuming that $f_{s}$ is very close to $f_{0}$ and letting $\Delta f=f_{0}-f_{s}$, (1) can be expressed as

$$
f(n)=\sin \left(2 \pi n+2 \pi \Delta f \frac{n}{f_{s}}\right)=\sin \left(2 \pi \Delta f \frac{n}{f_{s}}\right) .
$$




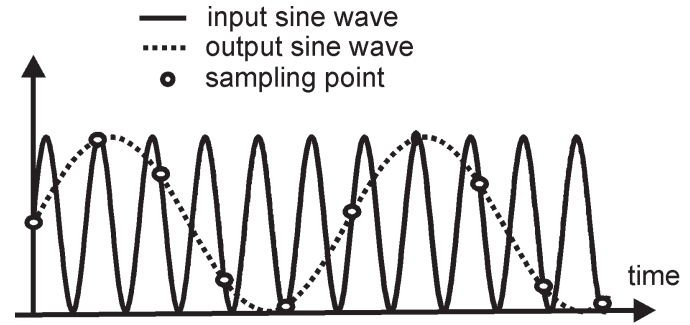

Fig. 5. Illustration of the subsampling concept.

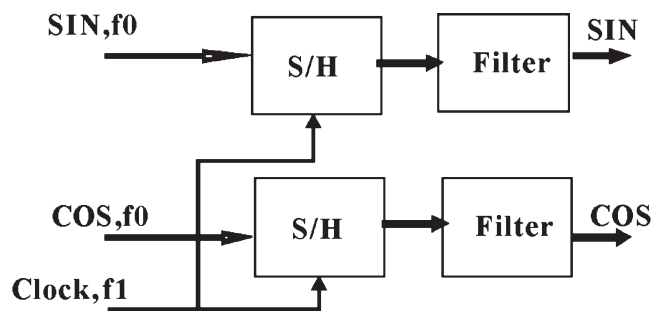

Fig. 6. Functional block diagram of the subsampling signal generator.

Compared with (1), (2) still represents a discrete sinusoid signal, but its frequency is $\Delta f$. This principle is graphically shown in Fig. 5, where the sinusoid input (solid line) is sampled (dots) by a frequency very close to the signal frequency and a sinusoid output signal (dashed line) can be formed. A functional block diagram for the circuit to implement this new subsampling signal generator scheme is shown in Fig. 6.

To achieve a constant frequency sinusoid output using subsampling, it is critical to maintain a constant frequency difference between the sampling and input signals. Thus, both the RCDAC updating clock and the subsampling clock have been based on the same $10-\mathrm{MHz}$ master clock, dividing them by factors of $M$ and $N$, respectively. This permits a stable and very well controlled relationship between the two frequencies.

\section{Frequency Range and Coverage Density}

In the $100-\mathrm{Hz}$ to $10-\mathrm{kHz}$ range, the sinusoid output frequency of the QSSG is controlled by setting the frequency of the RCDAC updating clock, which is derived by dividing the master clock. With the RCDAC OSR set to $64 \times$, the QSSG output frequency can be expressed as

$$
f_{O}=\frac{f_{M}}{64 M}
$$

where $f_{M}$ is the frequency of the master clock, and $M$ is the updating clock division factor. By setting $M$ from 16 to 1562 , the QSSG (with a 10-MHz master clock) can generate sufficient frequency points between $100 \mathrm{~Hz}$ and $10 \mathrm{kHz}$ for impedance characterization applications.

The output frequency of the subsampling QSSG is determined by

$$
f_{\text {out }}=f_{O}-f_{s}=\frac{f_{M}}{64 M}-\frac{f_{M}}{n}
$$

where $N$ is the subsampling division factor, and $f_{\text {out }}, f_{o}$, $f_{s}$, and $f_{M}$ are the frequency of the subsampling output, the RCDAC QSSG output, the sampling signal, and the master clock, respectively, as shown in Fig. 3. For a given $f_{M}$ and $M$,
TABLE I

Output Frequency and Signal Generator Settings

\begin{tabular}{|c|c|c|c|c|}
\hline $\begin{array}{c}f_{\text {out }} \\
\text { Output } \\
\text { frequency } \\
\text { (Hz) }\end{array}$ & $\begin{array}{c}f_{\boldsymbol{0}} \\
\text { RCDAC } \\
\text { frequency } \\
\text { (Hz) }\end{array}$ & $\mathbf{M}$ & $\mathbf{N}$ & $\begin{array}{c}\text { Output } \\
\text { source }\end{array}$ \\
\hline $\mathbf{1 0 0} \mathbf{1 0 k}$ & varies & $1562-16$ & N/A & RCDAC \\
\hline $\mathbf{1 0}-\mathbf{1 0 0}$ & $10 \mathrm{k}$ & 16 & $1025-1035$ & \multirow{2}{*}{ Sub-sampling } \\
\cline { 1 - 4 } $\mathbf{0 . 1}-\mathbf{1 0}$ & $1 \mathrm{k}$ & 156 & $9985-10085$ & \\
\hline $\mathbf{1 m}-\mathbf{0 . 1}$ & 100 & 1562 & $100033-104218$ & \\
\hline
\end{tabular}

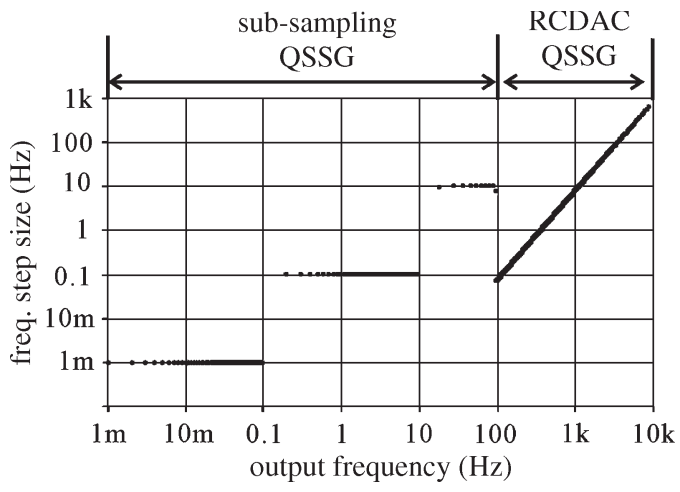

Fig. 7. Frequency step size over the operational frequency range.

if both $M$ and $N$ are integers, the minimum $f_{\text {out }}$ is achieved by setting $N=64 M+1$ and can be expressed by

$$
\begin{aligned}
f_{\text {out }, \text { min }}=f_{M}\left(\frac{1}{64 M}-\frac{1}{64 M+1}\right) \\
=\frac{f_{M}}{(64 M)(64 M+1)} \approx \frac{f_{O}^{2}}{f_{M}}
\end{aligned}
$$

which shows that, to lower the output frequency, either the master clock frequency can be increased or the output frequency of RCDAC signal generator can be decreased. However, $f_{o}$ cannot be very low for two reasons: First, similar to the DSP-based signal generator, the RCDAC signal generator with very low output frequencies would add significant complexity to the analog filter design because the sampling clock will be very close to the signal frequency. Second, because $f_{s}$ must be close to $f_{o}$, the sample-and-hold circuit would suffer significant leakage at a very low updating frequency. To avoid these problems, the lowest $f_{o}$ was set to $100 \mathrm{~Hz}$ in our implementation. Thus, to achieve a 1-mHz QSSG output frequency, (5) shows that the master clock should be $10 \mathrm{MHz}$.

Table I defines the RCDAC output frequency and the signal generator settings that will cover the desired $1-\mathrm{mHz}$ to $10-\mathrm{kHz}$ range. It also defines which signal source is directed to the output of the overall QSSG; from $1 \mathrm{mHz}$ to $100 \mathrm{~Hz}$, the subsampling block signal is output, and for $100 \mathrm{~Hz}$ to $10 \mathrm{kHz}$, the RCDAC generator signal is output.

Because $M$ and $N$ are integers, the possible output frequency values are discrete and cannot cover every point in the frequency range. However, in practice, continuous coverage is not necessary, provided the signal generator can maintain sufficient coverage density. The frequency step size at each frequency point is plotted in Fig. 7 and shows that at least ten points are produced per decade, which is more than enough for impedance measurement applications. 


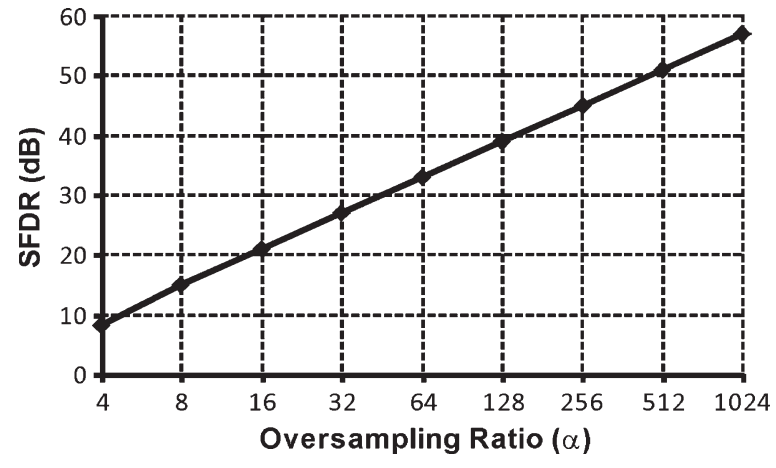

Fig. 8. Plot of the SFDR versus the OSR.

\section{VLSI REALIZATON}

\section{A. RCDAC for the High-Frequency QSSG}

The RCDAC QSSG generates quadrature sinusoid signals of $100 \mathrm{~Hz}$ and above. The most important parameter of the RCDAC, as functionally described in Fig. 4(b), is the number of taps along the resistor chain, which determines the OSR of the output sinusoid waveform. Although increasing the number of taps improves linearity, it increases switch control logic complexity and requires more silicon areas for the switches and routing metals.

To determine an optimal OSR, the output linearity of the DAC has been analyzed in terms of the spurious-free dynamic range (SFDR). The waveform of the RCDAC output is a sample-and-hold sine wave. Its typical spectrum includes the fundamental signal and sampling replicas at multiples of the sampling frequency, which are enveloped by a sinc function [14]. Only the first replica will be considered because it dominates the spurs. Thus, the SFDR is defined as

$$
\mathrm{SFDR}=20 \log \left(\frac{A_{0}}{A_{1}}\right)
$$

where $A_{0}$ and $A_{1}$ are the strengths of the fundamental signal and the first replica, respectively. Assuming the amplitude of the sample-and-hold sine wave to be 1

$$
\begin{aligned}
& A_{0}=\left|\frac{\alpha}{\pi} \sin \left(\frac{\pi}{\alpha}\right)\right| \\
& A_{1}=\sqrt{\left(\frac{\alpha}{\pi(\alpha-1)} \sin \left(\pi \frac{\alpha-1}{\alpha}\right)\right)^{2}+\left(\frac{\alpha}{\pi(\alpha+1)} \sin \left(\pi \frac{\alpha+1}{\alpha}\right)\right)^{2}}
\end{aligned}
$$

where $\alpha$ is the OSR at the DAC output. Based on (6)-(8), the relationship between SFDR and $\alpha$ can be plotted, as shown in Fig. 8. Because the replica strength is inversely proportional to the OSR, Fig. 8 shows that the SFDR is higher as the OSR increases.

Fig. 8 indicates that, when the OSR is greater than 512 , a linearity (SFDR) of more than $50 \mathrm{~dB}$ is achieved without replica suppression. However, this would require 256 or more taps on the DAC and is undesirable because of the required circuit complexity and chip area. Each tap needs a dedicated control signal; therefore, as the number of taps increases, so

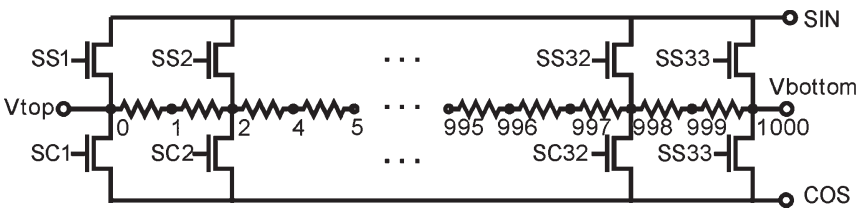

Fig. 9. Schematic of the RCDAC.

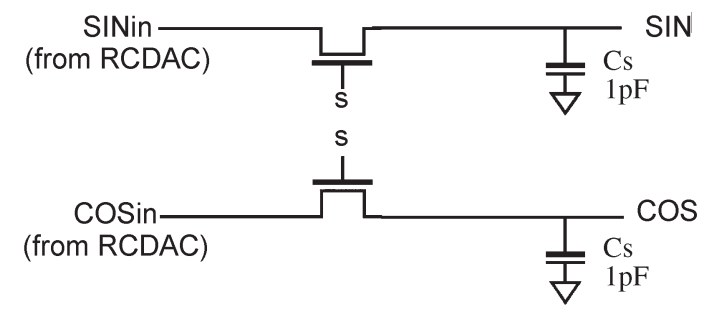

Fig. 10. T/H circuit for the subsampling QSSG.

does the required routing area. A preferable alternative is to lower the OSR and employ an LPF to further improve the SFDR by suppressing the replicas. However, the OSR cannot be too low because it determines the distance between the fundamental signal and its replicas, adding undesirable complexity to filter design. After analyzing this tradeoff to minimize the DAC and filter circuit complexity/area, the OSR was set to $64 \times$, requiring only 33 DAC taps. For a $64 \times$ OSR, (6)-(8) give an SFDR of $33 \mathrm{~dB}$; thus, the proceeding LPF needs to provide at least 17-dB suppression of the replicas to achieve a 50-dB SFDR. Compared with the alternative of using 256 taps and no filter, the routing area is decreased by $87 \%$. Because a simple compact second-order LPF is sufficient to provide 17-dB replica suppression, the chosen approach provides a significant overall area savings.

The schematic of the $64 \times$ OSR RCDAC is shown in Fig. 9 . For a high linearity, matching of DAC resistors is very important. In this sinusoidal tapped DAC, the resistances between taps are not equal, as they would be in a linear DAC. Because matching can best be achieved with same-size resistors, a resistor chain composed of 1000 same-size resistors was utilized, and the 33 tap points were sinusoidally mapped across the 1000 elements. At each tap point, a separate switch for sine and cosine taps was included. NMOS transistor switches were used instead of complimentary CMOS switches to simplify control logic and save chip area.

\section{B. T/H Circuit for the Subsampling $Q S S G$}

To sample and hold the sinusoid outputs of the RCDAC QSSG, the track-and-hold $(\mathrm{T} / \mathrm{H})$ circuit shown in Fig. 10 was realized. Minimum-sized NMOS transistors were employed for the switches for two reasons: First, charge injection of the switch is a potential problem for the simple $\mathrm{T} / \mathrm{H}$ circuit in Fig. 10. Having a small channel capacitance compared with the sampling capacitor (Cs), they minimize the charge injection effect. Second, they minimize the transistor source/drain leakage current to prevent a significant charge loss on the sampling capacitor, even at the lowest sampling frequency, $\sim 100 \mathrm{~Hz}$. Notice that, in this application, the sampling clock frequency is very close to the input reference sinusoid frequency, and therefore, the voltage change between two consecutive tracking 


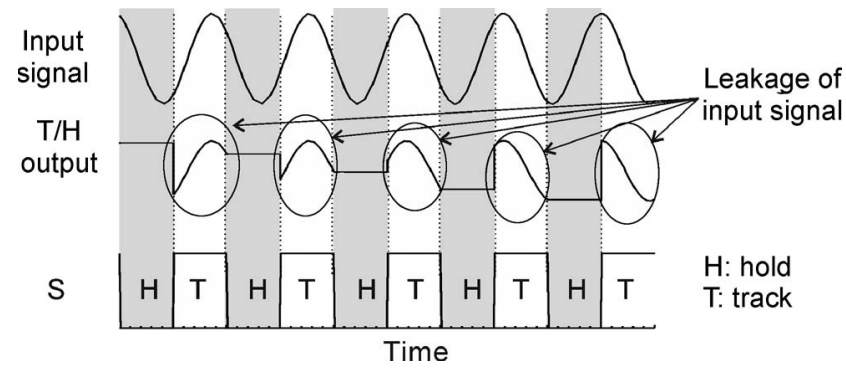

Fig. 11. Waveform of signals for the T/H system.

operations is very small. Therefore, even with a minimum-sized NMOS sampling switch, the signal will still quickly settle.

Another potential concern for the subsampling T/H system is pollution of the output signal during the tracking phase. The T/H system, as shown in Fig. 10, periodically switches between the $\mathrm{T} / \mathrm{H}$ modes based on the value of $S$, which is the switch control signal in Fig. 10. The desired low-frequency sinusoid output is composed of the sequence of hold values. However, in the track mode, the input and output are shorted, and therefore, the high-frequency input shows at the output. Thus, as shown in Fig. 11, the T/H system presents two time-multiplexed signals at its output, namely, the desired hold-mode output and the track-mode leakage. The duty cycle of $S$ determines the relative strength of these two signals. To achieve a high SFDR, the track-mode duration is set very narrow, around $100 \mathrm{~ns}$, much less than the $10-\mu \mathrm{s}$ or greater hold-mode duration, so that the track-mode leakage appears as glitches that are easily removed with a simple filter. An alternative solution to the track-mode leakage is to establish two paths, where, at any given time, one is in tracking mode and the other is in holding mode, and include an analog multiplexer that always connects the path in tracking mode to the output. However, this would double the number of switches connected to the sampling capacitor, increasing the source/drain leakage to potentially significant levels at the lowest sampling frequency $(\sim 100 \mathrm{~Hz})$.

\section{Filter Design}

To smooth the output and provide good replica suppression, the RCDAC QSSG requires an LPF. To cover its entire frequency range, a filter with selectable cutoff frequencies was determined to be most effective. The filter's cutoff frequency was set to $2 \mathrm{kHz}$ for output frequencies of $100 \mathrm{~Hz}$ to $1 \mathrm{kHz}$ and to $20 \mathrm{kHz}$ for $1-10 \mathrm{kHz}$. Note that, if coverage density were not critical, the RCDAC QSSG is capable of generating frequencies greater than $10 \mathrm{kHz}$ by setting the division factor $M$ to less than 16. To support this extended range, a filter cutoff frequency of $200 \mathrm{kHz}$ was also implemented.

At each of the three tunable settings, the least replica suppression occurs when the input is at the low end of the frequency range, where the replicas are the closest to the filter cutoff frequency. For example, when the cutoff frequency is $2 \mathrm{kHz}$, replicas are least suppressed when the output signal is at $100 \mathrm{~Hz}$. The worst-case replica suppression for the RCDAC tunable filter was designed to be $21 \mathrm{~dB}$, which is more than the $17 \mathrm{~dB}$ required to achieve the target $50-\mathrm{dB}$ SFDR with a 64-tap RCDAC.

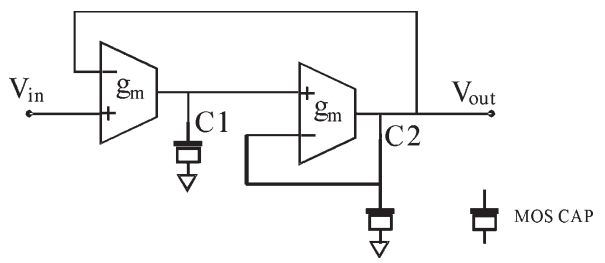

Fig. 12. Schematic of the $g_{m}-C$ biquadratic filter.

TABLE II

Output Frequency and Signal Generator Settings

\begin{tabular}{|c|c|c|c|c|}
\hline & $\mathrm{Gm}$ & $\mathrm{C} 1$ & $\mathrm{C} 2$ & $\begin{array}{c}\text { Cutoff } \\
\text { Frequency }\end{array}$ \\
\hline Fixed filter & $9 \mathrm{n}$ & $7.5 \mathrm{p}$ & $3.7 \mathrm{p}$ & 250 \\
\hline \multirow{2}{*}{$\begin{array}{c}\text { Tunable } \\
\text { filter }\end{array}$} & $60 \mathrm{n}$ & $5 \mathrm{p}$ & $2.5 \mathrm{p}$ & $2 \mathrm{k}$ \\
\cline { 2 - 5 } & $600 \mathrm{n}$ & $5 \mathrm{p}$ & $2.5 \mathrm{p}$ & $20 \mathrm{k}$ \\
\cline { 2 - 5 } & $600 \mathrm{n}$ & $0.5 \mathrm{p}$ & $0.25 \mathrm{p}$ & $200 \mathrm{k}$ \\
\hline
\end{tabular}

For the subsampling QSSG, notice that the sampling clock is very close to the RCDAC output frequency. Thus, the OSR is approximately the ratio between the RCDAC output frequency and the subsampling QSSG output frequency. Based on Table I, the OSR for outputs between $1 \mathrm{mHz}$ and $1 \mathrm{~Hz}$ is 1000 and above, which supports an SFDR of more than $50 \mathrm{~dB}$ (Fig. 8). Here, the filter is not required to provide replica suppression but will smooth the output of high-frequency switching glitches. Alternatively, for the $1-$ to $100-\mathrm{Hz}$ outputs, the OSR could be as low as 100, and additional 13-dB replica suppression is needed based on Fig. 8. The subsampling QSSG has a relatively constant sampling frequency because the output frequency is tuned by controlling the small frequency difference between the sampling and input reference frequencies. Thus, for output frequencies within $1-100 \mathrm{~Hz}$, the second-order replicas only appear at either $1 \mathrm{kHz}$ or $10 \mathrm{kHz}$, easing filter design requirements. An LPF with a fixed cutoff frequency of $250 \mathrm{~Hz}$ was determined to provide the necessary replica suppression.

For both the RCDAC and subsampling QSSG, a secondorder Butterworth filter was implemented to provide maximum passband flatness. The $g_{m}-C$ biquadratic circuit, as shown in Fig. 12 [15], was chosen to realize both the tunable and fixed cutoff frequency filters. To achieve Butterworth performance, the ratio of $C 1$ to $C 2$ was set to 2 so that $Q$ is fixed at 0.707 , which provides maximum flatness on the passband. The $g_{m}$ and $C$ values chosen for each of the filter configurations and settings are listed in Table II. Rather than using metal-insulator-metal capacitors, NMOS gate capacitances were used to minimize the chip area.

For the tunable filter, $g_{m}$ and the two capacitors each have been implemented with two selectable values, appropriately chosen to provide the three different cutoff frequencies shown in Table II. Both capacitors are implemented using two elements in parallel that can be switched on/off to set the correct value. For the fixed filter, with the capacitors set to reasonable on-chip values, a very small $g_{m}$ value $(9 \mathrm{nS})$ was needed to achieve the $250-\mathrm{Hz}$ cutoff frequency.

To generate $g_{m}$ for the filters, an operational transconductance amplifier (OTA) was used. The transconductance of the OTA should be small to match the small capacitances available on chip. There are several ways to design an OTA with a small $g_{m}$, including an OTA with current division [16] and/or source 
vdd

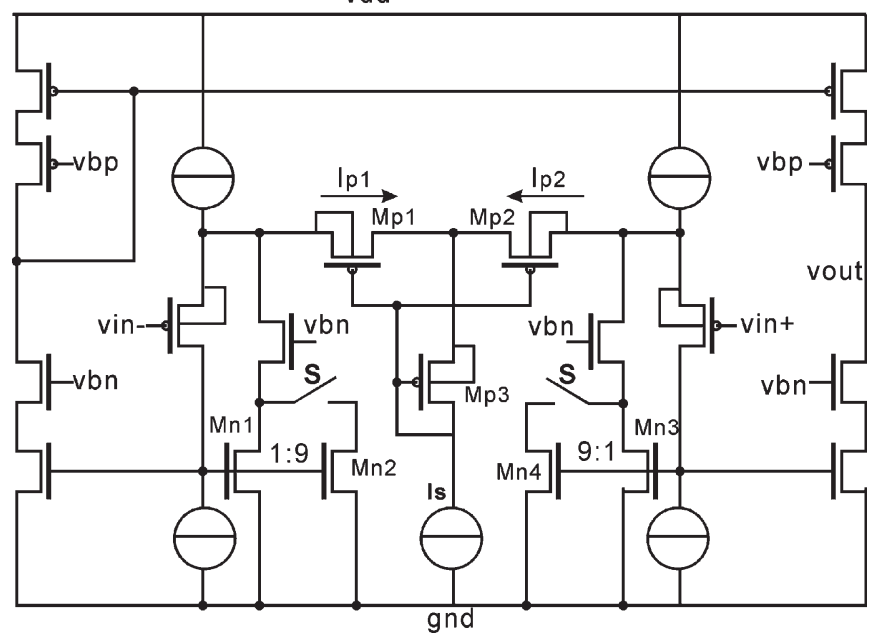

Fig. 13. Simplified schematic of the OTA.

degeneration [17], a floating-gate OTA [18], and a bulk-driven OTA [19]. Because an OTA with current division and source degeneration consumes less power and has an easily tunable $g_{m}$ value [20], this approach was selected for the QSSG filters. Fig. 13 shows the OTA that was designed to combine these two techniques in a new way. The input PMOS transistors are sized to $12 \mu \mathrm{m} / 1.2 \mu \mathrm{m}$. Current division was realized with the mirror structures Mn1 and Mn2, and Mn3 and Mn4. Internal feedback loops involving each of the input pair transistors control the gate of the current mirror transistors Mn1 and Mn3. With the current of the input transistors fixed, this structure gives good linearity performance. PMOS transistors (Mp1 and Mp2) working in a triode region were used as the source degeneration resistors, i.e., MOS-R. The $g_{m}$ of this OTA is

$$
\begin{aligned}
g_{M} & =K \frac{g_{O 1,2}}{2} \\
g_{O 1,2} & =\sqrt{2 \mu C_{\mathrm{OX}} \frac{W_{\mathrm{Mp} 1,2}}{L_{\mathrm{Mp} 1,2}} I_{s}}
\end{aligned}
$$

where $I_{S}$ is the bias current for the source degeneration MOS-R (Mp1 and Mp2), and $K$ is the ratio of current division, which is controlled by $S$ in Fig. 13. Thus, the transconductance $g_{m}$ can be tuned by the size of the MOS-R and its bias current.

For the filter with variable cutoff frequencies, the $W / L$ ratios of Mp1 and Mp2 were set to $1.5 \mu \mathrm{m} / 24 \mu \mathrm{m}$, and the bias current $I_{S}$ was set to $300 \mathrm{nA}$. With switch $S$ turned on or off, it can provide transconductances of 60 and $600 \mathrm{nS}$, respectively. For the filter with a fixed $250-\mathrm{Hz}$ cutoff frequency, the $W / L$ ratios of $\mathrm{Mp} 1$ and $\mathrm{Mp} 2$ were set to $1.5 \mu \mathrm{m} / 150 \mu \mathrm{m}$, and $I_{S}$ was set to $50 \mathrm{nA}$. With the switches $S$ always closed, a transconductance of $9 \mathrm{nS}$ was achieved.

\section{EXPERIMENTAL RESULTS}

The complete QSSG was implemented in a $0.5-\mu \mathrm{m}$ CMOS process and occupies $1 \mathrm{~mm} \times 1 \mathrm{~mm}$, as shown in Fig. 14. With a 3-V supply, the entire circuit draws only $60 \mu \mathrm{A}$. Test results demonstrate that it can generate the desired quadrature sinusoid signals from $1 \mathrm{mHz}$ and $10 \mathrm{kHz}$ with good matching and linearity. For the prototype circuit, external clock dividers were

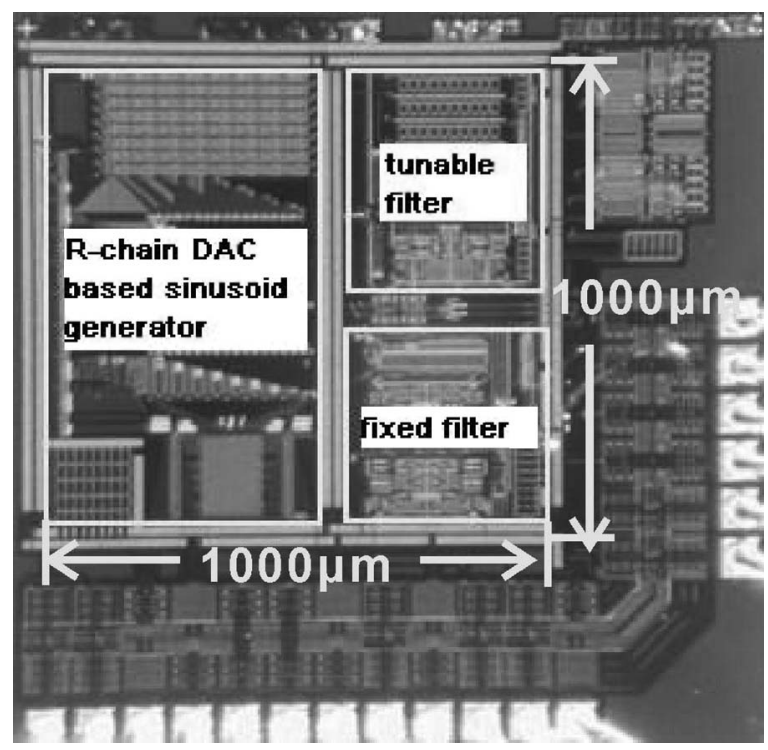

Fig. 14. Die photo of the new QSSG.

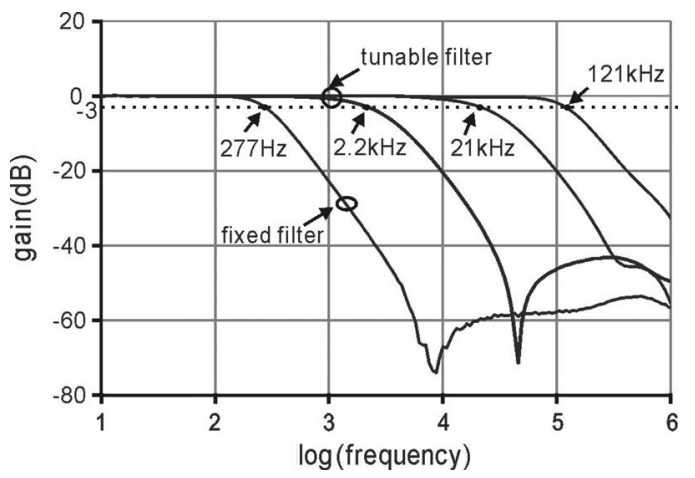

Fig. 15. Measured frequency response of the filters with $-3-\mathrm{dB}$ bandwidths labeled for each plot.

employed to generate the proper updating clock for the RCDAC and subsampling signal generator, as shown in Fig. 3. An integrated version of this divide-by- $N$ function could readily be achieved with digital counters or modular dividers [21].

\section{A. Analog Filter Characterization}

Because the performance of the analog filters is critical to the overall signal generator, they were independently tested. Experiments were performed to check the frequency transfer function and large signal linearity for the two second-order filters (one with three tunable cutoff frequencies, and the other with a fixed cutoff). Fig. 15 shows the transfer function of the filters combined into one plot for easy comparison. The measured cutoff frequencies are slightly shifted from design values, but they can still provide sufficient suppression of the replicas. To demonstrate the achieved filter linearity, Fig. 16 shows the output signal frequency spectrum for both the tunable and fixed filters. Only one plot is given for the tunable filter because the main nonlinearity contributors, namely, Mp1 and Mp2 in Fig. 13, are the same for all three cutoff frequencies. These plots show that both filters can provide more than $50-\mathrm{dB}$ linearity with up to a 500-mVpp sinusoid input. 

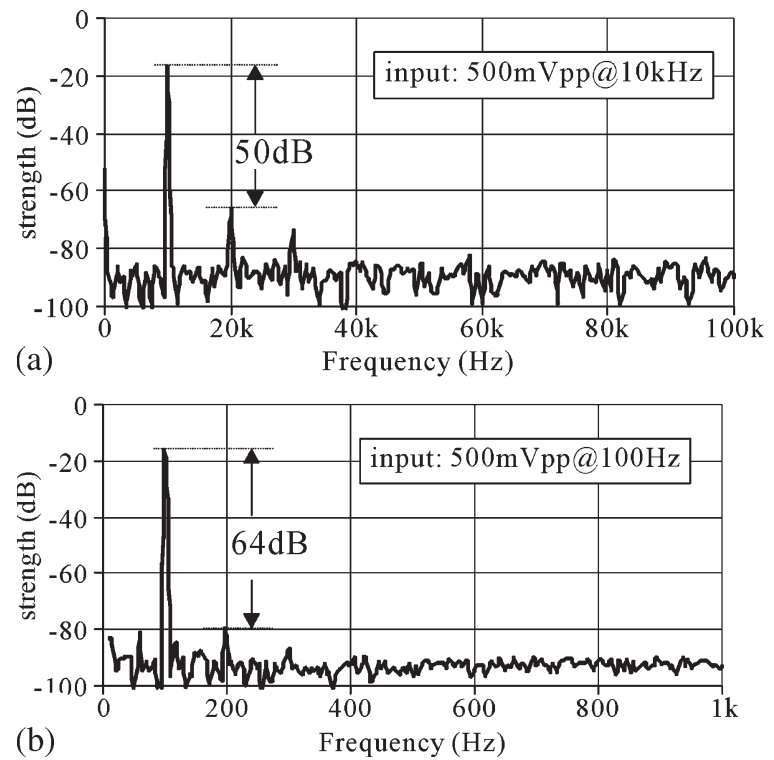

Fig. 16. Filter frequency spectrum demonstrating linearity. (a) Output of the tunable filter with $500 \mathrm{mVpp}$ at a $10-\mathrm{kHz}$ sinusoid input. (b) Output of the fixed filter with $500 \mathrm{mVpp}$ at a $100-\mathrm{Hz}$ sinusoid input.
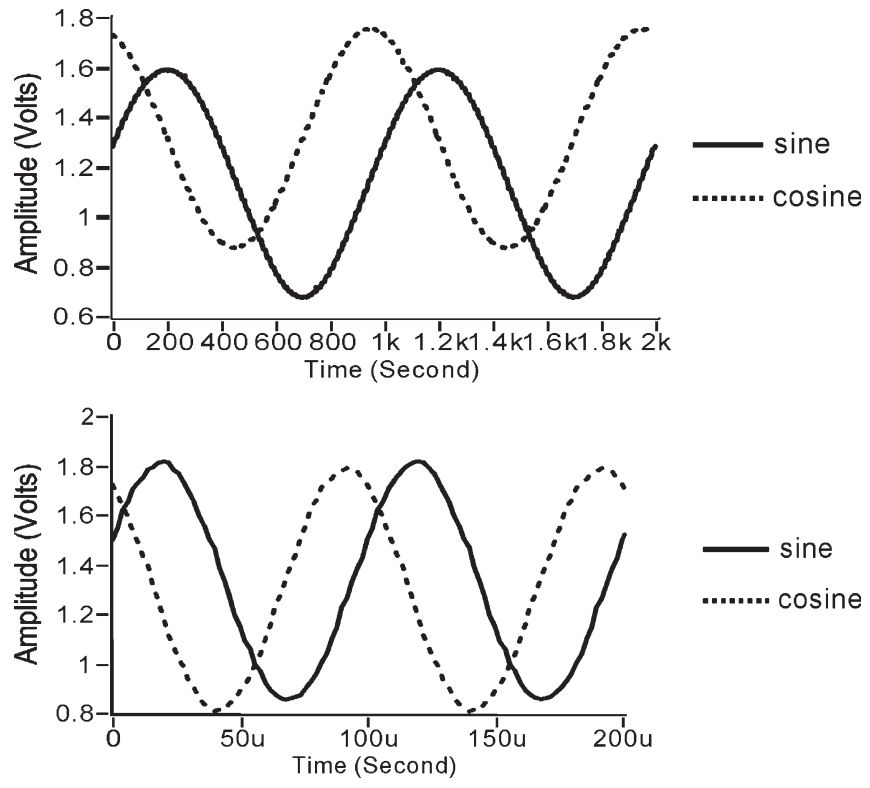

Fig. 17. Waveforms of quadrature sinusoid outputs at two frequencies: (top) $1 \mathrm{mHz}$ and (bottom) $10 \mathrm{kHz}$.

\section{B. Quadrature Sinusoid Output}

Several output frequency test points, ranging from $1 \mathrm{mHz}$ to $10 \mathrm{kHz}$, were generated and observed to be correct. The waveforms of two extreme output frequencies are plotted in Fig. 17. Fig. 17 shows a direct-current offset between $1-\mathrm{mHz}$ sine and cosine outputs. This is partially due to the offset in the fixed filters and is constant over frequency for all subsampling circuit $(1 \mathrm{mHz}$ to $100 \mathrm{~Hz}$ ) outputs, allowing it to be precalibrated.

To quantify how well the sine and cosine results match expectations, the phase mismatch (variation from $90^{\circ}$ between sinusoid and cosine outputs), amplitude mismatch, and total harmonic distortion (THD) were measured across the entire frequency range. These results, which are plotted in Figs. 18-20,

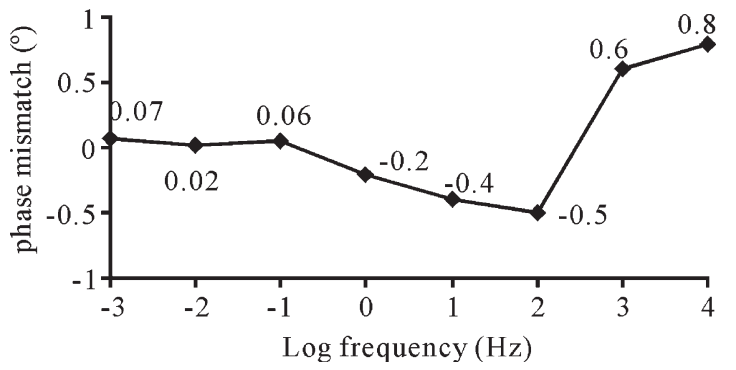

Fig. 18. Phase mismatch of the output quadrature signals over frequency.

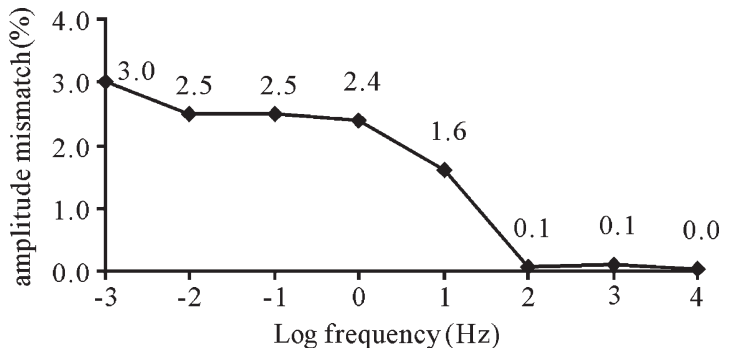

Fig. 19. Amplitude mismatch of the output quadrature signals over frequency.

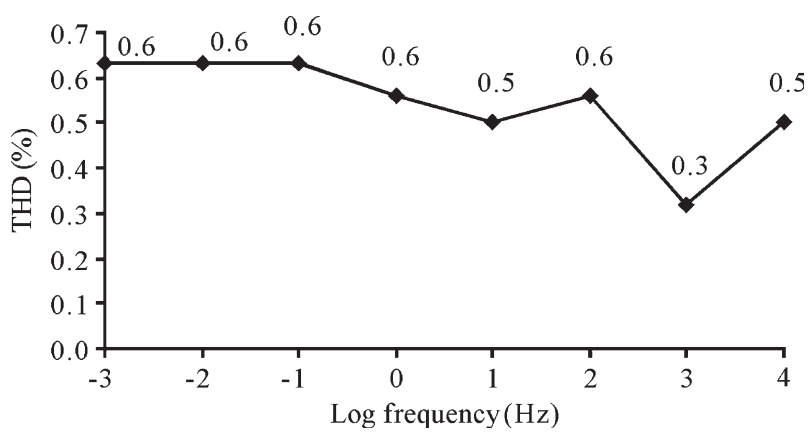

Fig. 20. THD of the output quadrature signals.

TABLE III

PERFormance SUMMARY OF THE QSSG

\begin{tabular}{|l|l|}
\hline Technology & $0.5 \mu \mathrm{m}$ CMOS \\
\hline Die area & $1 \mathrm{~mm}^{2}$ \\
\hline Power consumption & $60 \mu \mathrm{A}$ at $3 \mathrm{~V}$ \\
\hline Frequency range & $1 \mathrm{~m} \mathrm{~Hz}-10 \mathrm{kHz}$ \\
\hline Phase mismatch & $<0.8^{\circ}$ \\
\hline Amplitude mismatching & $<3 \%$ \\
\hline THD & $<0.6 \%$ \\
\hline
\end{tabular}

respectively, show that the new QSSG design can provide highquality quadrature sinusoid outputs with good phase $\left(<0.8^{\circ}\right)$ and amplitude matching $(<3 \%)$ between sine and cosine outputs. Frequency accuracy is inherently guaranteed by digital control of the clock frequencies. The measured THD is smaller than $0.6 \%$ over the entire seven-order frequency range. Performance is summarized in Table III.

\section{CONCLUSION}

An on-chip QSSG with a seven-order frequency span $(1 \mathrm{mHz}$ to $10 \mathrm{kHz}$ ) suitable for miniaturized impedance measurement instruments has been presented and described. To our knowledge, this signal generator achieves the widest frequency range reported to date. Utilizing a hybrid architecture that employs the 
subsampling technique in an original manner, the overall QSSG provides excellent phase $\left(0.8^{\circ}\right)$ and amplitude $(3 \%)$ matching between quadrature sinusoid signals and less than $0.6 \%$ THD over its entire $1-\mathrm{mHz}$ to $10-\mathrm{kHz}$ span. It inherently guarantees frequency-tuning accuracy with purely digital control. The new QSSG circuit was implemented in a $0.5-\mu \mathrm{m}$ CMOS process, where it occupies only $1 \mathrm{~mm}^{2}$ and consumes only $60 \mu \mathrm{A}$ at $3 \mathrm{~V}$. Measurement results confirm that the design meets the frequency range and performance requirement for on-chip sensor impedance instrumentation.

\section{REFERENCES}

[1] M. Min, O. Martens, and T. Parve, "Lock-in measurement of bioimpedance variations," Measurement, vol. 27, no. 1, pp. 21-28, Jan. 2000.

[2] F. Giess, M. G. Friedrich, J. Heberley, R. L. Naumann, and W. Knoll, "The protein-tethered lipid bilayer: A novel mimic of the biological membrane," Biophys. J., vol. 87, no. 5, pp. 3213-3220, Nov. 2004.

[3] R. Naumann, D. Walz, S. M. Schiller, and W. Knoll, "Kinetics of valinomycin-mediated $\mathrm{K}^{+}$ion transport through tethered bilayer lipid membranes," J. Electroanal. Chem., vol. 550/551, pp. 241-252, Jul. 2003.

[4] Product Specification for SPPOSC Series, Frequency Devices, Inc., Ottawa, IL. [Online]. Available: http://www.freqdev.com/products/osc/ spposc.pdf

[5] Product Specification for AFG3000 Series, Tektronix, Inc., Richardson, TX. [Online]. Available: http://www.tek.com/products/signal_sources/

[6] Understanding Signal Generation Methodologies, Tektronics, Inc., Richardson, TX. [Online]. Available: http://www2.tek.com/cmsreplive/ tirep/4477/2006.09.20.08.10.50_4477_EN.pdf

[7] I. A. Khan and S. Khwaja, "An integrated $g_{m}-C$ quadrature oscillator," Int. J. Electron., vol. 87, pp. 1353-1357, Nov. 2000.

[8] B. Linares-Barranco, T. Serrano-Gotarredona, J. Romas-Martos, J. Ceballos-Caceres, J. Mora, and A. Lnares-Barranco, "A precise $90^{\circ}$ quadrature OTA-C oscillator tunable in the $50-130-\mathrm{MHz}$ range," IEEE Trans. Circuits Syst. I, Reg. Papers, vol. 51, no. 4, pp. 649-664, Apr. 2004

[9] T. Serrano-Gotarredona and B. Linares-Barranco, "7-decade tuning range CMOS OTA-C sinusoidal VCO," Electron. Lett., vol. 34, no. 17, pp. 1621-1622, Aug. 1998.

[10] S. Franco, Design With Operational Amplifier and Analog Integrated Circuits, 3rd ed. New York: McGraw-Hill, 2002.

[11] Z. Tang, O. Ishizuka, and H. Matsumoto, "MOS triangle-to-sine wave convertor based on subthreshold operation," Electron. Lett., vol. 26, no. 23, pp. 1983-1985, Dec. 1990.

[12] B. Mesgarzadeh, M. Hansson, and A. Alvandpour, "Jitter characteristic in charge recovery resonant clock distribution," IEEE J. Solid-State Circuits, vol. 42, no. 7, pp. 1618-1625, Jul. 2007.

[13] R. B. Staszewskl, C. M. Hung, K. Magglo, J. Wallberg, D. Leipol, and P. T. Balsara, "All-digital phase-domain TX frequency synthesizer for Bluetooth radios in $0.13 \mu \mathrm{m}$ CMOS," in IEEE Int. Conf. Solid State Circuits, San Francisco, CA, 2004, pp. 268-527.

[14] D. A. Johns and K. Martin, Analog Integrated Circuit Design. Hoboken, NJ: Wiley, 1997.

[15] R. L. Geiger and E. Sánchez-Sinencio, "Active filter design using operational transconductance amplifiers: A tutorial," IEEE Circuits Devices Mag., vol. 1, no. 2, pp. 20-32, Mar. 1985.

[16] J. Silva-Martínez and S. Solís-Bustos, "Design considerations for highperformance very-low-frequency filters," in IEEE Int. Symp. Circuits Syst., San Francisco, CA, 1999, pp. 648-651.
[17] P. Garde, "Transconductance cancellation for operational amplifiers," IEEE J. Solid-State Circuits, vol. SSC-12, no. 3, pp. 310-311, Jun. 1977.

[18] L. Yin, S. H. K. Embabi, and E. Sánchez-Sinencio, "A floating-gate MOSFET D/A converter," in IEEE Int. Symp. Circuits Syst., San Francisco, CA, 1997, pp. 409-412.

[19] R. Fried and C. C. Enz, "Bulk-driven MOS transconductor with extended linear range," Electron. Lett., vol. 32, no. 7, pp. 638-640, Mar. 1996.

[20] A. Veeravalli, E. Sanchez-Sinencio, and J. Silva-Martines, "Transconductance amplifier structures with very small transconductances: A comparative design approach," IEEE J. Solid-State Circuits, vol. 37, no. 6, pp. 770-775, Jun. 2002.

[21] C. S. Vaucher, I. Ferencic, M. Locher, S. Sedvallson, U. Voegeli, and Z. Wang, "A family of low-power truly modular programmable dividers in standard 0.35- $\mu$ s CMOS technology," IEEE J. Solid-State Circuits, vol. 35 , no. 7 , pp. 1039-1045, Jul. 2000

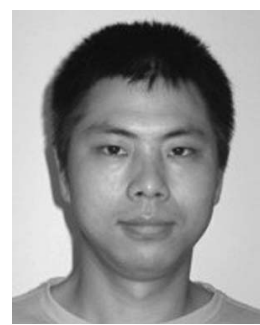

Chao Yang received the B.S. and M.S. degrees in electrical engineering from Tsinghua University, Beijing, China, in 1999 and 2002, respectively, and the Ph.D. degree in electrical engineering from Michigan State University, East Lansing, in 2008.

$\mathrm{He}$ is currently with Marvell Semiconductor, Inc., Santa Clara, CA. His research focus is the analog/mixed-signal IC design for the biomedical and biochemical instrumentation microsystem.

Dr. Yang was the recipient of the Excellence Scholarship from Tsinghua University from 1996 to 1998 and of the VLSI/SOC Fellowship from Michigan State University in 2007. He was also the recipient of the Third Place Award (out of 40 participants) in both phase 1 and phase 2 of the Semiconductor Research Corporation/Semiconductor Industry Association (SRC/SIA) SoC Design Challenge.

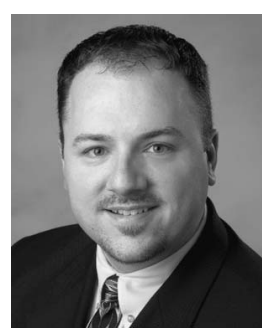

Andrew J. Mason (S'90-M'99-SM'06) received the B.S. degree (with highest distinction) in physics from Western Kentucky University, Bowling Green, in 1991, the B.S.E.E. degree (with honors) from Georgia Institute of Technology, Atlanta, in 1992, and the M.S. and Ph.D. degrees in electrical engineering from The University of Michigan, Ann Arbor, in 1994 and 2000, respectively.

From 1999 to 2001, he was an Assistant Professor with the University of Kentucky, Lexington. In 2001, he joined the Department of Electrical and Computer Engineering, Michigan State University, East Lansing, where he is currently an Associate Professor. His research addresses many areas of mixed-signal circuit design and the fabrication of integrated microsystems. His current projects include compact low-power bioelectrochemical interrogation circuits, adaptive chemical sensor interface circuits, post-CMOS fabrication of electrochemical sensor arrays, and mixed-signal integrated circuits for signal processing in neural implants.

Dr. Mason serves on the Sensory Systems and Biomedical Circuits and Systems Technical Committees of the IEEE Circuits and Systems Society and on the Technical Program Committee for the IEEE International Conference on Sensors. He was the recipient of the Michigan State University Teacher-Scholar Award in 2006. 